\title{
An Energy Aware Multipath Routing Algorithm for Wireless Sensor Networks
}

\author{
https://doi.org/10.3991/ijoe.v13i04.7061 \\ Liping LV \\ Jiaozuo University, Henan, China \\ e0690248guaao766@163.com
}

\begin{abstract}
Wireless sensor network is a new field of computer science and technology research. It has a very broad application prospects. In order to improve the network survival time, it is very important to design efficient energyconstrained routing protocols. In this paper, we studied the characteristics of wireless sensor networks, and analyzed the design criteria of sensor network routing algorithms. In view of the shortcomings of traditional algorithms, we proposed an energy-aware multi-path algorithm. When selecting a data transmission path, the energy-aware multi-path algorithm can avoid nodes with low energy levels. At the same time, it takes the remaining energy of the node and the number of hops as one of the measures of the path selection. The multi-path routing algorithm realized the low energy consumption of the data transmission path, thus effectively prolonging the network lifetime. Compared with the traditional algorithm, the results show that our method has high reliability and energy efficiency.
\end{abstract}

Keywords—multi-path, routing algorithm, wireless sensor network

\section{Introduction}

Wireless sensor network is a new technology [3]. At present, it is still in the research stage [4]. Network layer routing protocol is one of the difficult problems in wireless sensor networks, and also one of the factors that affect the performance of the whole network [1]. Therefore, the network design is critical to the routing protocol. Because of the different application background of wireless sensor network, it is more complex to design a general routing protocol. In general, for different application background, we use different methods to design communication protocols. Compared with the traditional wireless network, the wireless sensor network has its particularity. At the same time, the application of wireless sensor networks and the general communication network has a significant difference. Therefore, the traditional wireless network routing protocol cannot be directly applied to wireless sensor networks [2].

With the development of micro-electromechanical systems and communication technology, wireless sensor networks have been used in many fields [5]. In the mili- 
tary field, wireless sensor networks can be used to build a battlefield command system that combines functions such as command, control, communication, intelligence, surveillance, detection and location [6]. In the commercial field, wireless sensor networks can achieve intelligent home environment. This will provide people with more comfortable, convenient and user-friendly smart home and office environment. In other commercial areas, such as factory automation production lines, warehousing management, intelligent transportation, etc. also introduced the technology [8]. In the field of medical and health care, it can achieve remote real-time monitoring of the patient's physical condition [7]. Sensor nodes can monitor the body's various physiological conditions for a long time. By using the sensor network, we can understand the patient's condition at any time. In the field of environmental monitoring and protection, wireless sensor networks can be used to achieve the ecological environment monitoring, volcanic eruptions monitoring, forest fire alarm monitoring and so on. In the field of special environmental monitoring, the sensor network also has its unique technical advantages [9].

Faced with these practical applications, it is important to ensure reliable transmission of data. Due to the restrictions of cost and volume, sensor nodes typically use batteries to provide energy. However, there are a large number of nodes deployed throughout the network, and the working environment is poor. Therefore, it is very difficult to replace the battery of the node. In general, there are few researches on wireless sensor networks in china. The study of new technologies has great strategic significance for the whole society and the national economy.

\section{State of the Art}

At present, some research scholars have proposed a variety of different application targets based on the routing protocol. They solve various routing problems in wireless sensor networks in different ways.

Mahesh K. and Marina et al. proposed a multi-path routing protocol. The protocol is a routing protocol that proposed in the network [11]. It is a routing mechanism that improves the reliability of data transmission by establishing multiple links between data source nodes and sink nodes. However, the reliability of data transmission has not been significantly improved when selecting the shortest fixed route to transmit data.

Deepak Ganesan et al. proposed a new multi-path establishment method, which is called entangled multipath [10]. Compared to the previous classic disjoint multi-path method, it can achieve higher transmission reliability. The method is to set up an alternate node for each point on the main path of the source node to the destination node. When a node on the primary path fails, it can immediately use the alternate node for transmission. Although the winding multi-path considering the balance between the energy consumption of multiple paths, but due to the limited number of 
spare paths, winding multi-path cannot be very good to meet the needs of reliable data transmission.

De $S$ and others proposed a method to improve the reliability of data transmission by using multipath [12]. In the first few jumps, the data is quickly expanded into multiple paths, which avoids the failure to start the transmission. The expanded data is transmitted to the base station along a single path. Unlike Braided, which only sends low rate data to maintain alternate paths, MESH transfers data over multiple paths. As a result, MESH achieves high reliability, but it consumes more energy.

S. Dulman et al. proposed the use of multipath routing to improve the reliability of sensor networks. This algorithm is useful for data transmission in unreliable environments [13]. However, this technology will greatly increase the flow. Thus, there is a compromise between network traffic and reliability. This compromise uses a redundant function that depends on the degree of multipath and the probability of available paths. The idea is to divide the source packet into several subpackages, and then send each subpackage through one of its multiple available paths. The simulation confirms that the source packet can be re-restored even if some subpackets are lost.

China's sensor network research is still relatively small, but some colleges and universities and research institutions have been actively carried out on the sensor network research. For example, the National Natural Science Foundation funded the "energy-efficient sensor network protocol research" and so on. Now, the wireless sensor network research has been included in the national "Eleventh Five-Year" plan. It is one of the important research directions of wireless sensor network routing protocol to improve the reliability of data transmission and to extend the network lifetime.

\section{Methods}

\subsection{Energy saving strategy analysis of routing algorithm}

The routing protocol is responsible for routing generation and route selection. It needs to consider how to pass data traffic from the source node to the destination node globally. For the whole wireless sensor network, the energy efficiency of the network layer routing protocol is an important way to save energy in the wireless sensor network. The important question is how to find an energy-efficient multi-hop route between the source node and the destination node. Routing protocols often use different routing metrics to improve energy efficiency. On the basis of common routing protocols, energy-efficient routing algorithms take into account the relevant energy consumption factors, and introduce new routing metrics related to energy consumption, so as to achieve energy savings. In general, energy efficient routing protocol research focuses on two areas: minimizing the total energy consumed by routing communications and maximizing network lifetime.

The minimum communication energy routing protocol tries to find a path with minimum energy consumption between the source node and the destination node. 
Assuming that a packet is passed through nodes $n_{1} \ldots . . n_{i}, n_{i+1}, \ldots, n_{k}$, we use $\mathrm{E}_{\mathrm{Tx}}(\mathrm{a}, \mathrm{b})$ to represent the energy consumed by the hop-by-hop transfer packet from node a to node $b$. Then, if the received energy is not taken into account, the total energy consumed by the primary transmission of the packet is:

$$
\mathrm{E}=\sum_{\mathrm{i}=1}^{\mathrm{k}-1} \mathrm{E}_{\mathrm{Tx}}\left(\mathrm{n}_{\mathrm{i}}, \mathrm{n}_{\mathrm{i}+1}\right)
$$

The goal of this protocol is to minimize the total energy $\mathrm{E}$ of a packet.

The minimize the communication energy strategy algorithm is simple, and it can be well grafted to the original on-demand routing strategy. However, it contains two shortcomings: On the one hand, this type of energy-saving strategy may make some key nodes frequently fall into the minimum transmission power path, and a large number of loads will make these nodes premature energy depletion, resulting in network differentiation. On the other hand, it generates a lot of hop, which increases endto-end latency, and it will result in a large number of retransmissions on poor wireless links. The simulation results show that the recalculation of the consumption in some cases even more than the strategy itself to reduce energy consumption.

The energy-saving routing protocol that maximizes the network lifetime takes into account the fact that the traffic load is distributed between the nodes, balancing the energy consumption of the nodes in the network, thus delaying the segmentation of the network and maximizing the lifetime of the entire network. At present, the researchers have proposed a lot of maximum network survival time routing algorithms and protocols, such as MBCR and MMBCR and so on [10].

MBCR strategy from the global network to get the average battery energy sufficient route, but it still cannot avoid some of the nodes in the routing of the battery energy consumption faster, leading to premature failure. The strategy avoids the nodes with the smallest battery energy in the network as much as possible when selecting the route, thus realizing the fair use of the node. However, the average life of the nodes will be reduced by the choice of those with greater energy consumption.

We give the two measures of energy-saving routing algorithm to consider the indicators: minimizes the total energy consumed by routing communications and maximizes the lifetime of the network. We can combine these two metrics to minimize the total energy consumed by routing communications while maximizing network life time.

\subsection{Network model}

As the design goals of wireless sensor networks are very different, we make the following assumptions about the wireless sensor networks applicable to the algorithm: 
1. The wireless sensor network consists of a base station and n sensor nodes, and the base station and the node are fixed.

2. The energy of the base station is sufficient, the sensor node energy is limited, and it cannot be replaced. In order to ensure the consistency of the energy of the nodes, each node has the same initial energy. Each node can get its own energy level at any time.

3. Each node in the network has a unique number, and the transmit power is fixed and cannot be adjusted. Nodes do not need to know their location information. Therefore, the node does not need to be equipped with GPS devices.

4. There is no unidirectional link between the nodes in the network, that is, the A node can communicate with the $\mathrm{B}$ node, and the $\mathrm{B}$ node can communicate with the A node.

\subsection{Selection rule of sub - optimal path of EMRA algorithm}

When the primary path fails, it is necessary to select one of the alternate paths to continue transmitting data as a secondary path. The rules for selecting the suboptimal path of the EMRA algorithm are as follows: through the information in the data packet, the source node can know the node dependency between the established paths. In the rest of the alternate path set, we use the associated main path as the secondary path. The priority path is selected by definition only if the path of the alternate path set does not have the same number of nodes or shared nodes in the main path.

Through an example, we understand the rules of suboptimal path selection. As shown in Figure 1, in the network topology, we use ERMA algorithm. Assume that there are three paths between source node $S$ and destination node $D$, which are path $C$ (S-E-F-G-C-D), path H (S-A-B-G-H-D), path Z (S-X-B-Y-Z-D), respectively. From the received packets, in the path set of the source node to the destination node, we can know that path $\mathrm{C}$ and path $\mathrm{H}$ have shared node $\mathrm{G}$, and path $\mathrm{H}$ and path $\mathrm{Z}$ have shared node $\mathrm{B}$.

1. If the source node chooses path $\mathrm{C}$ as the main path, when the path $\mathrm{C}$ causes the path to break due to the failure of the node, the source node avoids the path $\mathrm{H}$ of the common node, and selects the path $\mathrm{C}$ as the secondary path according to the suboptimal path selection rule. Similarly, if the path $\mathrm{Z}$ is selected as the primary path, the source node selects path $\mathrm{C}$ as the secondary path when the primary path fails.

2. If the source node chooses path $\mathrm{H}$ as the main path, when path $\mathrm{H}$ is off, there is a common node for path $\mathrm{C}$, path $\mathrm{Z}$ and path $\mathrm{H}$, then the suboptimal path is selected according to the definition. 


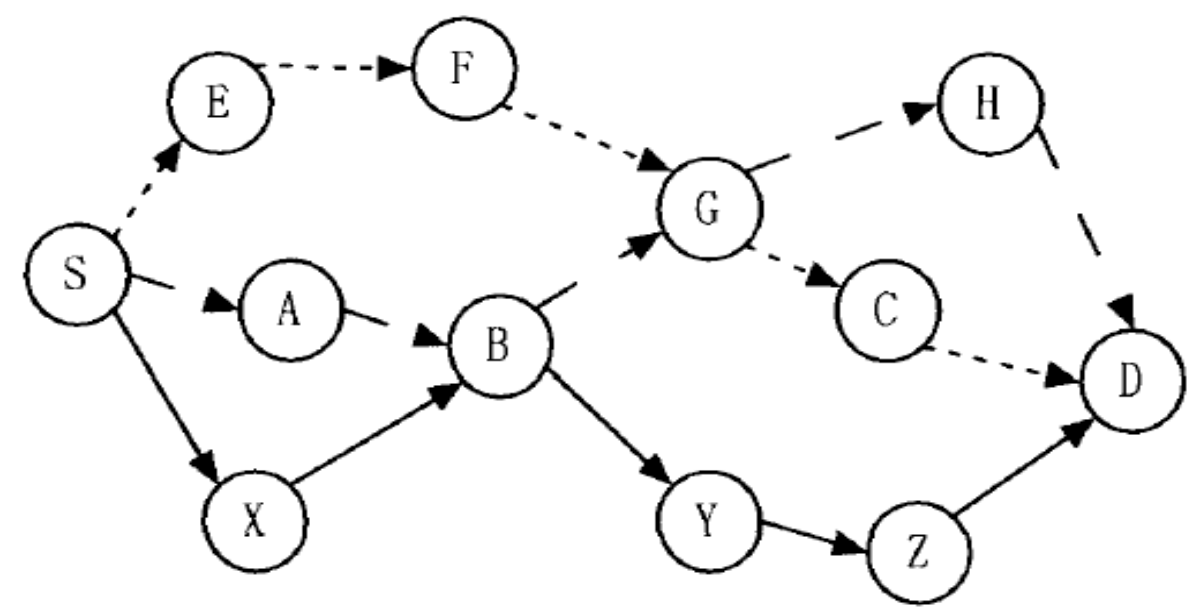

Fig. 1. Node topology diagram

The selection rule of the suboptimal path ensures that when the primary path fails, the source node can preferentially select the path with the least number of shared nodes as the secondary path. It reduces the probability of path chain failure, and enhances the network's fault tolerance.

\section{$4 \quad$ Experiment and result analysis}

\subsection{The selection of parameters}

Through its network of effective sensor nodes, wireless sensor networks to perform environmental monitoring and data collection tasks. However, the validity of a sensor node depends on whether the activity of the node can monitor the acquisition data and the connectivity with other nodes in the network, that is, whether it can send and forward the monitoring data to the convergence node. With the increase of network running time, the sensor nodes become effective nodes due to energy exhaustion or connectivity. When the number of active nodes in the network accounts for less than a certain percentage of all nodes, the lifetime of the sensor network is considered to be ended.

As there is no unified measurement standard for routing protocols in wireless sensor networks, according to the sensor network routing protocol performance measurement method, we mainly verify the energy efficiency and transmission reliability of the algorithm. From the two aspects of the network survival time and the average packet delivery rate, we conducted a comparative analysis.

Network survival time: The performance evaluation criteria mainly measure the trend of network survival time under different algorithms. It mainly includes the number of nodes surviving and the average residual energy in two aspects. 
Network node survival rate: this criterion is used to compare algorithms to protect nodes with less residual energy from premature depletion. In the simulation process, the node with the residual energy value below the energy needed to receive the data packet is defined as the dead node. The standard reflects the status of nodes in a network at a time, and is used to reflect the length of network lifetime.

The average residual energy value of the node: this standard indicates the average energy consumption of each node in the network, which is used to reflect the average energy of each node in the network, and to reflect the length of the network lifetime from one side.

The average delivery rate of the group: The average delivery rate is defined as the ratio of the number of application layer data packets received by the sink node and the number of packets sent by the application layer of the source node in the network simulation process. It is the main measure to reflect the reliability of data transmission. It can be calculated by using Equation 2. The higher the average delivery rate of the packet, the less the packet lost in the transmission process, and the better the network performance.

Group average delivery rate $=$ number of successful packets received $/$ number of packets sent

\subsection{Simulation results and performance analysis}

The survival time of the network is the effective time of the network, which is the embodiment of the practical value of the network. This measure reflects the direct effect of the network energy consumption problem, that is, it has the same meaning as the whole network energy consumption index of the network.

The survival rate of the network node: Network node survival can be defined in three forms: first node died, half node died and last node died. To a certain extent, the node's death time reflects the energy balance in the network. In the comparison process, if the first node dead time is short, it indicates that the wireless sensor network in the process of collecting data communication too much use of a certain number of sensor nodes for data transmission. If the death time of the first node is relatively long, it indicates that the energy consumption in the data submission process is more evenly distributed on more nodes in the network. To a certain extent, it increases the viability and effectiveness of the network. The network survival time comparison of EMRA algorithm and AOMDV protocol, DD protocol, MDP protocol is shown in Figure 2. In the figure, the ordinate is the number of surviving nodes in the network, and the abscissa is the number of rounds of data transmission. After the network is initialized, the node periodically sends data, and when a node is exhausted, it exits the network and simulates the operation until all nodes die.

As can be seen from Figure 2, at the beginning of the network operation, there was no difference in the percentage of network nodes in the four algorithms. However, with the increase in network running time, this difference appears and gradually become apparent. AOMDV, DD and MDP began to die at 135,140,138, respectively, 
and EMRA began node death in 148 cycles. The half of the EMRA node's death time is 1.2 times of the AOMDV node. The half of the EMRA node's death time is 1.08 times of the DD node. The half of the EMRA node's death time is 1.12 times of the MDP node. The death time of all EMRA nodes is 1.31 times of AOMDV. The death time of all EMRA nodes is 1.09 times of DD. The death time of all EMRA nodes is 1.18 times of MDP. From the experimental results, we can see that it significantly extends the network's survival time.

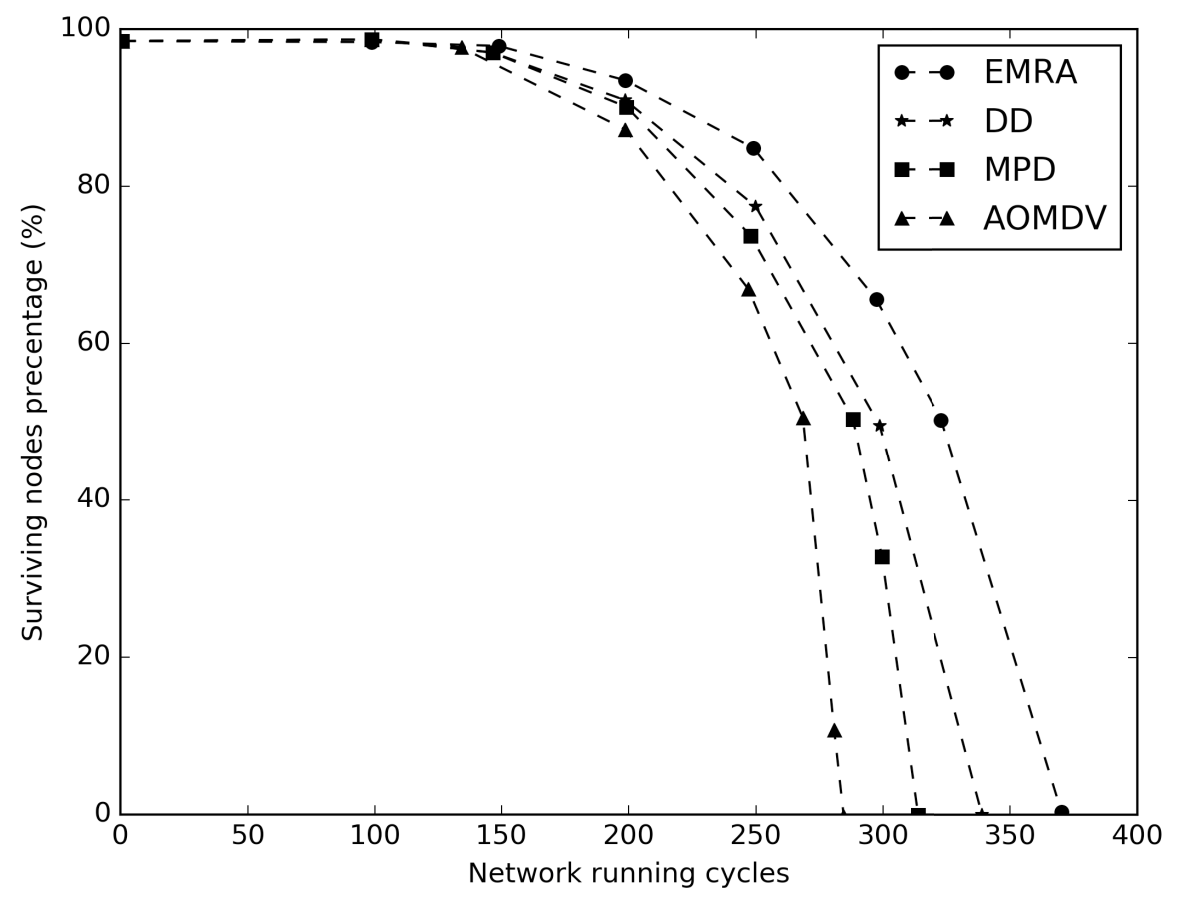

Fig. 2. The comparison curves of the number of surviving nodes

The average residual energy of the node: Figure 3 shows the simulation comparison of the average residual energy of the nodes of the four routing algorithms. In the figure, the ordinate is the average residual energy of the node, and the abscissa is the number of network running cycles. As shown in Figure 3, there was no significant difference in the performance of the four algorithms in the average residual energy of the nodes before 150 cycles of the initial operation of the network. However, with the increase in network running time, the performance of this difference is gradually obvious. From the whole period of network operation, the average residual energy of the EMRA algorithm is slower than the other three algorithms. This indicates that it has a high energy efficiency, and it makes the loss of energy more evenly distributed to all nodes. Therefore, from the perspective of the average residual energy of the nodes, we further verify that the network running the algorithm can achieve a longer network lifetime. 


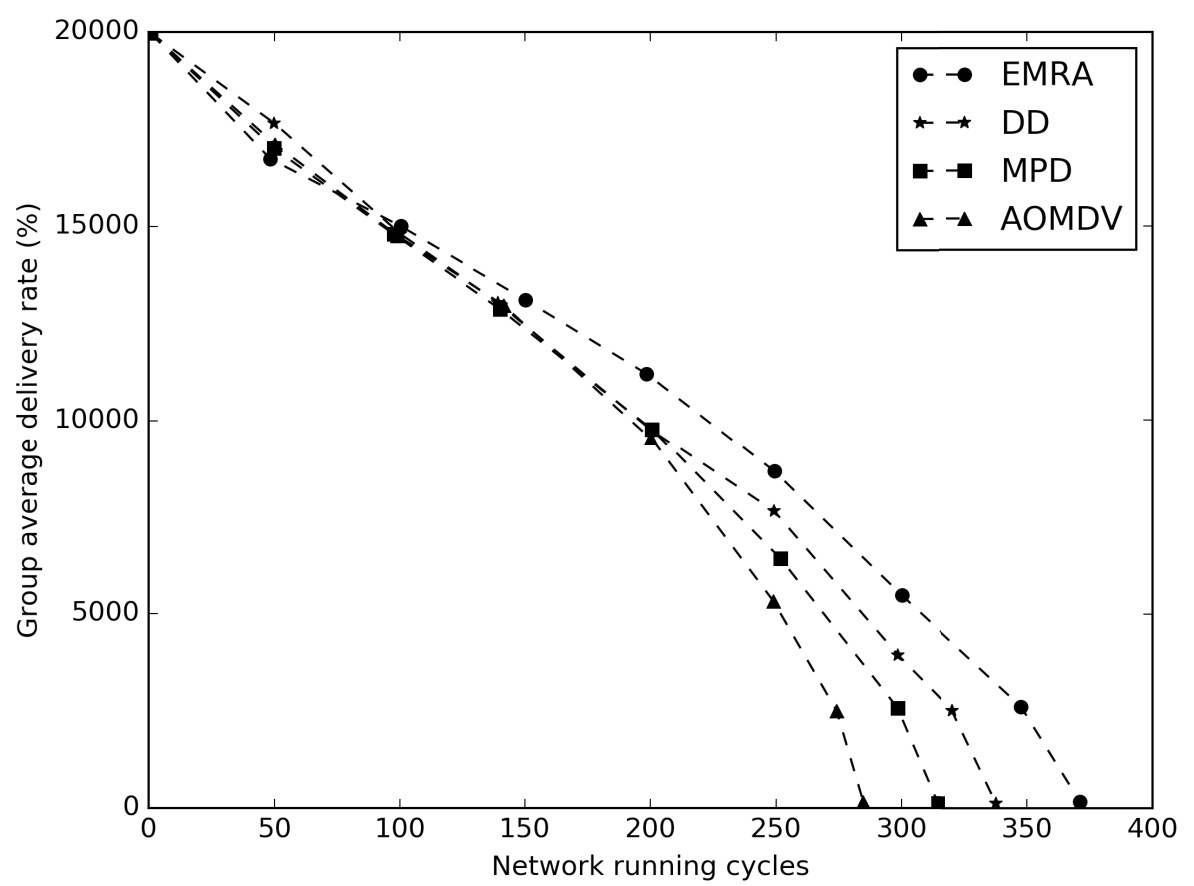

Fig. 3. The comparison curves of the average residual energy of the node

\subsection{Group average delivery rate}

In this simulation, it is mainly to measure the ratio of the number of data packets successfully received by the sink node to the number of packets sent by the source node under different algorithms. This reflects the reliability of data transmission. Figure 4 is the group average delivery rate with time curve. In the figure, the ordinate is the average delivery rate of the group, and the abscissa is the number of cycles to complete the network operation. In the same experimental environment, the average delivery rate of the four algorithms is not the same. In the whole period of the network operation, the EMRA algorithm has got a better result, and it shows a high transmission reliability.

Optimizing the data transmission path, improving the efficiency of node energy and extending the lifetime of the network is the main goal of wireless sensor network routing protocol design. It can be seen from the simulation results that the algorithm has high data transmission reliability. It can improve the energy efficiency and energy balance, thus effectively prolonging the lifetime of the whole network. 


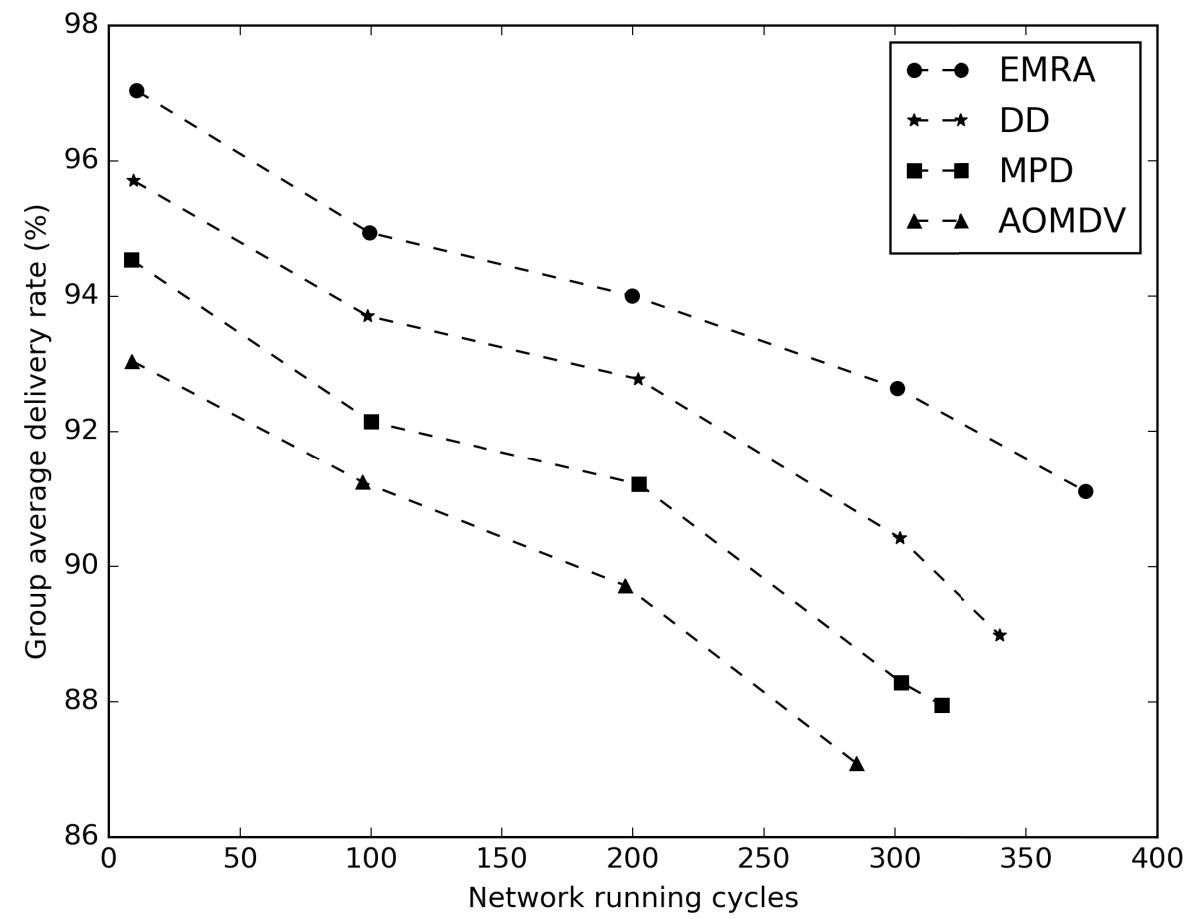

Fig. 4. The comparison curves of the group average delivery rate

\section{Conclusion}

In order to improve the energy efficiency and extend the survival time of the sensor network, we analyzed the stability of the multi-path routing. Based on the traditional multipath algorithm, we propose an energy-aware multipath algorithm. When selecting the main path, the algorithm uses the residual energy and the intermediate hop count as one of the metrics for the path selection. At the same time, it avoids the nodes with low energy level, so as to achieve the purpose of balancing the network energy consumption and prolonging the network survival time. When the main path is broken, the source node can preferentially select the path that is not related to the main path as a new data transmission path, thus enhancing the network fault tolerance. Compared with the traditional multi-path routing algorithm, the results show that the energy-aware multipath routing algorithm has high data transmission reliability and energy efficiency. 


\section{References}

[1] Anwar, A., Sridharan, D., Anwar, A., \& Sridharan, D. (2015). A survey on routing protocols for wireless sensor networks in various environments. International Journal of Computer Applications, 112(5), 13-29.

[2] Guravaiah, K., \& Velusamy, R. L. (2015). Rfdmrp: river formation dynamics based multihop routing protocol for data collection in wireless sensor networks. Procedia Computer Science, 54, 31-36. https://doi.org/10.1016/j.procs.2015.06.004

[3] Hao, J., Yao, Z., Huang, K., Zhang, B., \& Li, C. (2016). A gradient-based multiple-path routing protocol for low duty-cycled wireless sensor networks. Wireless Communications \& Mobile Computing, 16(5), 538-549. https://doi.org/10.1002/wcm.2552

[4] He, D., Mujica, G., Portilla, J., \& Riesgo, T. (2015). Modelling and planning reliable wireless sensor networks based on multi-objective optimization genetic algorithm with changeable length. Journal of Heuristics, 21(2), 257-300. https://doi.org/10.1007/s10732-0149261-2

[5] Hu, Y. F., Ding, Y. S., Ren, L. H., Hao, K. R., \& Han, H. (2015). An endocrine cooperative particle swarm optimization algorithm for routing recovery problem of wireless sensor networks with multiple mobile sinks. Information Sciences, 300, 100-113. https://doi.org/10.1016/j.ins.2014.11.052

[6] Karthick, K. ,., \& Kumar, R. V. (2015). An energy-saving routing algorithm for wireless body sensor network using data compression technique. International Journal of Computer Applications, 114(15), 26-29. https://doi.org/10.5120/20055-2020

[7] Kumari, N., Gupta, D. K., \& Kumar, M. (2015). Improved teen routing protocol with multi hop and multi path in wireless sensor networks. International Journal of Computer Applications, 129(7), 11-16. https://doi.org/10.5120/ijca2015906783

[8] Mann, P. S., \& Singh, S. (2017). Energy-Efficient Hierarchical Routing for Wireless Sensor Networks: A Swarm Intelligence Approach. Wireless Personal Communications, 92(2), 785-805. https://doi.org/10.1007/s11277-016-3577-1

[9] Naranjo, P. G. V., Shojafar, M., Mostafaei, H., Pooranian, Z., \& Baccarelli, E. (2016). Psep: a prolong stable election routing algorithm for energy-limited heterogeneous fogsupported wireless sensor networks. Journal of Supercomputing, 1-23.

[10] Rajasoundaran, S., Narayanasamy, P., \& Riasudheen, H. (2016). A Hybrid Secure Routing and Monitoring Mechanism Using Authorized and Concealed Arbitrary Watchdogs in Wireless Sensor Network. Intelligent Systems And Communication (NCISC-2016), 177. https://doi.org/10.5958/2249-7315.2016.00664.x

[11] Rezaei, E., Baradaran, A. A., \& Heydariyan, A. (2016). Multi-hop routing algorithm using steiner points for reducing energy consumption in wireless sensor networks. Wireless Personal Communications, 86(3), 1557-1570. https://doi.org/10.1007/s11277-015-3006-x

[12] Yigit, M., Gungor, V. C., Fadel, E., Nassef, L., Akkari, N., \& Akyildiz, I. F. (2016). Channel-aware routing and priority-aware multi-channel scheduling for WSN-based smart grid applications. Journal of Network and Computer Applications, 71, 50-58. https://doi.org/10.1016/j.jnca.2016.05.015

[13] Zhu, R., Zhao, Y., Yang, H., Yu, X., Zhang, J., Yousefpour, A., ... \& Jue, J. P. (2016). Dynamic time and spectrum fragmentation-aware service provisioning in elastic optical networks with multi-path routing. Optical Fiber Technology, 32, 13-22. https://doi.org/10.1016/j.yofte.2016.08.009 
Paper-An Energy Aware Multipath Routing Algorithm for Wireless Sensor Networks

\section{Author}

Liping LV is with Jiaozuo University, Henan, China (e-mail: e0690248guaao766@163.com).

Article submitted 26 March 2017. Published as resubmitted by the author 28 April 2017. 